\title{
Characterization and structural properties of bamboo fibre solid foams
}

\author{
H. Malekzadeh • N. S. B. Md Zaid • E. Bele $(\mathbb{D}$
}

Received: 5 August 2020/Accepted: 29 October 2020/Published online: 16 November 2020

(C) The Author(s) 2020

\begin{abstract}
In this work, cellulose fibres extracted from bamboo culms were used to fabricate two types of cellular materials: rigid foams and fibrous networks. A relatively simple and low-technology fabrication method is presented, using natural binders and blowing agents to manufacture rigid foams, and fibrillation by partial hydrolysis in $\mathrm{H}_{2} \mathrm{SO}_{4}$ to manufacture fibrous networks. The compressive response is related to the internal microstructure and processing parameters. In the case of fibrous networks, the achievable relative density range is determined by the length of initial fibres and extent of external fibrillation. The compressive properties are dictated both by the density of the network and strength of the fibrous bridges, showing a linear stiffness-density relationship due to the length of fibres, and an inverse relationship at increased external fibrillation. The rigid foams showed an orthotropic internal microstructure but nearly isotropic compressive response, due to the influence of the interpenetrating void structure on the deformation and fracture mechanisms. The results show the potential of bamboo-fibre porous materials as low cost, lightweight structural materials.
\end{abstract}

H. Malekzadeh · N. S. B. Md Zaid · E. Bele $(\bowtie)$

Department of Mechanical Engineering, University

College London, Torrington Place, London WC1E 7JE, UK

e-mail: e.bele@ucl.ac.uk
Keywords Cellular solids - Bamboo fibre · Structural properties - Compressive strength . Microstructure $\cdot$ Biodegradable

\section{Introduction}

Lightweight cellular solids, e.g. foams and fibrous networks, find wide use in weight-sensitive applications. Their ability to sustain large deformations in compression (Miltz and Ramon 1990), and the large volume fraction of enclosed air, makes them particularly useful in energy absorption applications (Gibson and Ashby 1997), insulation from thermal and vibrational sources (Poehler et al. 2017), filters, and architectural panels (Härkäsalmi et al. 2017).

Continuous efforts are being made towards the manufacturing of environmentally-friendly counterparts to the traditional plastic and metal foams, with particular emphasis on two fronts: (1) the biodegradability and life-cycle impact of the constituent materials, and (2) the energy consumption of the material extraction and foaming processes. Foaming techniques for networks of natural fibres date to the early 1960s, e.g. in the formation of foam-formed pulp sheets, where suspensions of natural fibres were stabilised with surfactants and drained of the liquid phase (Radvan 1964; Bernardin 1969; Chung 1974). More recently, progress has been made on developing 
structurally competitive biopolymer foams from several types of organic materials. These involve the usage of vegetable oils (Tu et al. 2008), and wood byproducts such as lignin (Mimin et al. 2016; Tondi et al. 2016; Stevens et al. 2010) and tannin (Szczurek et al. 2014; Tondi and Pizzi 2009; Merle et al. 2016).

Cellulose is the most abundant organic polymer sourced from plant cell walls (Sfiligoj et al. 2013); this characteristic and its compatibility with other materials has led to its fast-growing application as a precursor in foam-making, commonly termed as cellulose-based foams. Examples include fibre foams, where cellulose fibres are suspended in a binder of a secondary phase (Svagan et al. 2011; Ottenhall et al. 2018; Saz-Orozco et al. 2013), which is subsequently blown with a foaming agent, and dried using freeze or critical point drying. In such architectures, the fibres are dispersed in cell walls, and connected at the cell wall or nodal junctions (Alimadadi et al. 2018). A second class of porous architectures are cellulose fibre networks, usually created through the fibre-foaming technique: fibres are suspended in a liquid foam, stabilised by surfactants, and the liquid is drained e.g. (Demitri et al. 2014; Rapp et al. 2014; Kang et al. 2012; Alimadadi and Uesaka 2016; Cervin et al. 2013; Kinnunen et al. 2013; Madani et al. 2014; Ketoja et al. 2019; Ferreira and Rezende 2018). These structures produce a network-like microstructure with random fibre orientation, and the fibres are connected by mechanical interlocking or chemical bonds.

The sustainability of cellulose cellular solids depends largely on the primary source of fibres, and the energy consumption of the extraction and foaming processes. Often, the former receives little attention, however it is an important consideration both in resource consumption (i.e. proportion of fibres extracted from a given mass of organic material), and time scale for the regeneration of the primary sources. Challenges related to deforestation and uncertain availability of wood fibres make the sourcing and extraction of cellulose fibres crucial for responsible industrial manufacturing (Correia et al. 2015).

In this study, we use simple manufacturing methods to create low-density cellular solids from bamboo fibres, a source that has not been considered previously in the literature of cellulose foams. The manufacturing methods aim to minimise energy consumption through low temperatures and simple techniques: foams are fabricated with natural binders, surfactants and blowing agents, and fibrous networks are fabricated without either. Bamboo is a very sustainable source of cellulose fibres: its primary constituents are cellulose, hemicellulose and lignin (approx. 90\% of its composition). It possesses a high volume fraction of cellulose fibres (approx. $48 \%$ by mass (Moradbak et al. 2015)) compared to e.g. wood (39-45\% by mass (Mohamed and Hassabo 2015)) and grass (33-45\% by mass (Prasad et al. 2007)). Furthermore, unlike wood sources which are in high demand for other uses and take take a relatively long time between harvests, bamboo can be reharvested every three years without detrimental environmental impacts (Harris and Koomson 2015).

This work describes the full process of fabricating cellular solids from bamboo culms. The fibres are used to create two types of solids: a fibrous material (with natural binding from external fibrillation) and a rigid close-celled foam. For the fibrous networks, the fibre length and extent of external fibrillation are related to the mechanical properties and achieved density. For the rigid foams, the effect of morphological anisotropy on mechanical properties is investigated. This paper is organised as follows. First the effect of the fibre extraction treatment and partial fibrillation is reported on the surface morphology of fibres. Next the microstructure (cell aspect ratio, fibre mean free span) and density of the cellular solids is connected to the processing parameters (initial fibre length, extent of fibrillation). Finally, the compressive response is related to the governing deformation and failure mechanisms.

\section{Materials and methods}

Cellulose fibre extraction

Moso bamboo culm was used as the main source of fibres in this study. The culm nodes were discarded to ensure consistency of the initial fibre content; the remaining internodal parts of the culms were crushed and ground in a high speed grinder. The ground particles were subsequently sieved in three size ranges: $500-850 \mu \mathrm{m}, 850-1000 \mu \mathrm{m}$, and $1000-1500$ $\mu \mathrm{m}$. The cellulose fibres were isolated from noncellulosic content through three processes: dewaxing, delignification, and mercerisation. Dewaxing was 
carried out using the Leavitt-Danzer method (Leavitt and Danzer 1993). A 2:1 solution of toluene and ethanol, heated to $250{ }^{\circ} \mathrm{C}$, was passed through a Soxhlet extractor column containing the bamboo particles. The extraction process was continued for 5-6 cycles, until no colour remained in the extraction column. Upon completion, the dewaxed particles were rinsed with the toluene-ethanol solution, and dried at $70{ }^{\circ} \mathrm{C}$ for $2 \mathrm{~h}$.

Delignification is a process that dissolves lignin and hemicellulose. Dewaxed fibres were mixed into a solution of $35 \mathrm{wt} . \%$ hydrogen peroxide, glacial acetic acid, and titanium(IV) oxide catalyst. The solution was heated at $130{ }^{\circ} \mathrm{C}$ for $2 \mathrm{~h}$, and subsequently rinsed with distilled water to achieve a neutral $\mathrm{pH}$, before being dried in an oven at $70^{\circ} \mathrm{C} \mathrm{C}$ for $2 \mathrm{~h}$. Mercerisation is a finishing treatment that strengthens fibres and further removes non-cellulosic constituents. The delignified fibres were incorporated into a solution of $6 \mathrm{wt} \%$ sodium hydroxide, and heated at $75{ }^{\circ} \mathrm{C}$, while being agitated at $150 \mathrm{rpm}$ for $2 \mathrm{~h}$. Finally, the solution was again filtered using a Buchner funnel and subsequently rinsed and dried. To ensure that maximal non-cellulosic constituents are disposed, this process was repeated two to three times until white, soft cellulose fibre clumps were obtained.

\section{Foaming process}

Two types of cellular solids were manufactured: a fibrous network and a close-celled foam. The fibrous network was created by partial hydrolysis of the fibres in $\mathrm{H}_{2} \mathrm{SO}_{4}$, after a similar process used for Eucalyptus pulp (Ferreira and Rezende 2018). The extracted fibres were dispersed in distilled water, stirred to homogenise the suspension, and mixed at $45{ }^{\circ} \mathrm{C}$ with $\mathrm{H}_{2} \mathrm{SO}_{4}$ solutions of concentrations $20-65 \% \mathrm{v} / \mathrm{v}$. Maintaining the temperature, the mixture was stirred at $600 \mathrm{rpm}$ for $1 \mathrm{~h}$ and then left to cool to room temperature. Subsequently, the suspension was centrifuged five times at $5000 \mathrm{rpm}$ for $10 \mathrm{~min}$, removing the supernatant and rinsing the fibres with deionised water after every step, until the supernatant was clear and neutral $\mathrm{pH}$ was achieved. Finally, the dispersion was added into bottomless cylindrical stainless steel moulds, dried at $60{ }^{\circ} \mathrm{C}$ for $12 \mathrm{~h}$, and cured at room temperature for two days.

To prepare the close-celled foams, a pulp slurry with consistency of $2 \mathrm{wt} . \%$ was prepared by soaking the obtained bamboo cellulose fibres in solution of water and $1 \%$ sodium hydroxide for $24 \mathrm{~h}$. The formed pulp was stirred at speed of $1600 \mathrm{rpm}$ for $15 \mathrm{~min}$, and $2.5 \mathrm{wt} \%$ of sodium bicarbonate was added to the mixture as a blowing agent. Equal amount of water was also added to the mixture to activate this blowing agent in the reaction. The pulp was stabilized by addition of of 0.5 wt.\% guar gum as binder. The resulting mixture was set to agitate at speed of 1600 rpm for $15 \mathrm{~min}$, then $2.5 \mathrm{wt} \%$ sodium dodecyl sulphate was added as surfactant. To increase the rigidity of the solid foam, $2 \mathrm{wt} . \%$ MDI was added in the mixture. Finally, the foam mixture was drained in bottomless cylindrical moulds and cured in room temperature for 2 days.

Characterisation of morphology and mechanical testing

Fourier Transform Infrared Spectroscopy (FTIR) was used to identify differences in chemical composition of untreated and treated (delignified) fibre clusters, to evaluate the extent of removal of non-cellulosic constituents. The spectra were obtained with a PerkinElmer Spectrum Two spectrometer within the wavenumber range of $4000-400 \mathrm{~cm}^{-1}$. The surface morphologies of fibres and foams were characterised with a Hitachi S-3400N scanning electron microscope.

Uniaxial compression tests were conducted on in accordance with the ASTM D1621 standard (ASTM D1621-16, 2016). Cubic specimens with edge length of $5 \mathrm{~cm}$ were machined with flat edges for both types of foams, and tested in an Instron 5985 universal testing system equipped with a $500 \mathrm{~N}$ load cell and deflectometer. Quasi-static tests were conducted at a strain rate of $10^{-3} \mathrm{~s}^{-1}$. The rigid foams were tested in the three principal directions to assess the influence of morphologic anisotropy, using at least 5 specimens/ test.

\section{Morphology of fibres and solid foams}

Fibre morphology

An important objective of fibre extraction from natural materials is the removal of extraneous phases, and successful separation of fibre bundles. In bamboo stalks, cellulose fibres must be extracted from their 
binders: lignin, hemicellulose, waxes and pectin. FTIR spectra of fibres before and after the cellulose extraction treatments (dewaxing, delignification mercerisation) are shown in Fig. 1. The spectra are normalised at the prominent peak of $1030 \mathrm{~cm}^{-1}$, which corresponds to $\mathrm{C}-\mathrm{O}$ bond stretching in primary alcohol groups, present in cellulose and hemicellulose (Fabiyi and Ogunleye 2015). Two other peaks that are universally present are those at $900 \mathrm{~cm}^{-1}$, produced by the $\mathrm{C}-\mathrm{H}$ bond stretching vibration in the glucose rings of cellulose (Fabiyi and Ogunleye 2015), and at 3330 $\mathrm{cm}^{-1}$, attributed to O-H hydroxyl groups in cellulose structure (Faix 1991). The presence of the above peaks indicates that the cellulose content remained intact throughout the treatment. The medium peaks near $1700 \mathrm{~cm}^{-1}$ in untreated fibres correspond to carbonyl stretching of acetyl and ester groups in hemicellulose (Fabiyi and Ogunleye 2015); the absence of these peaks in the spectrum of treated fibres indicates the removal of hemicellulose. Characteristic peaks of lignin are at $1420 \mathrm{~cm}^{-1}$ and $1500 \mathrm{~cm}^{-1}$, due to the vibration of the $\mathrm{C}=\mathrm{C}$ bonds in the aromatic skeleton, and $\mathrm{C}-\mathrm{H}$ bond asymmetric stretching respectively (Fabiyi and Ogunleye 2015; Reddy et al. 2012). Their absence in the spectra indicates removal of lignin. Finally, the intensity peak at $1237 \mathrm{~cm}^{-1}$ corresponds to the $\mathrm{C}-\mathrm{O}$ bond stretching in the acetyl groups of hemicellulose and lignin(Fabiyi and Ogunleye 2015) — this peak is also absent in the spectrum of treated fibres.

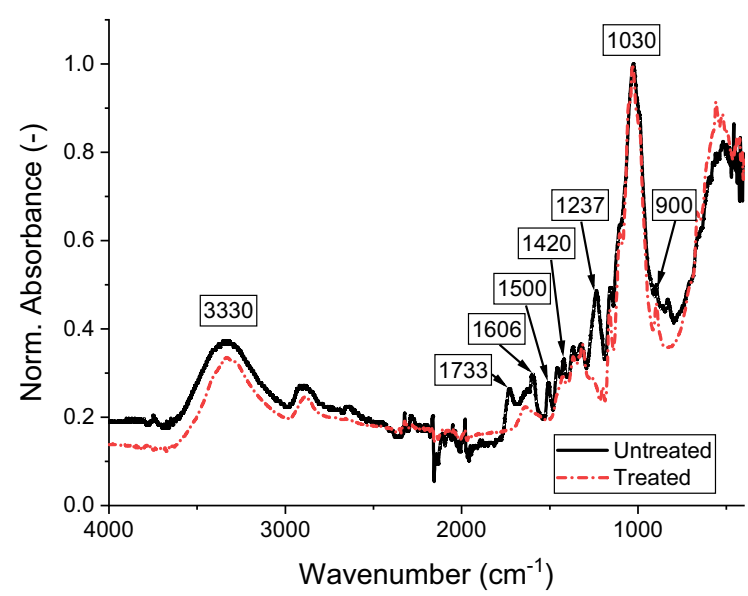

Fig. 1 FTIR spectra (normalised absorbance vs. wavenumber) of bamboo fibres before and after dewaxing, delignification and mercerisation treatments
The morphology of as-treated fibres is shown in Fig. 2a. The treatment processes, particularly delignification, largely dissolves the binder phases, allowing fibre bundles to disintegrate. Some non-cellulosic components remain, apparent by intact bundles of fibres, highlighted by arrows in Fig. 2a. There is also evidence of globular structures assigned to lignin agglomerates (Lima et al. 2013). The area fraction of undissolved fibre bundles (normalised by the total area of fibres) was obtained through image analysis; this indicated that $<10 \%$ of the obtained fibres were in unseparated clumps. The separated fibres, shown in Fig. 2b, exhibit a rough surface of multiple valleys and troughs; this morphology is typical of cellulose fibres that have been stripped of the non-cellulosic surrounding layers (Johar et al. 2012). A similar surface morphology has been shown in fibres extracted by only alkaline treatments, e.g. using $\mathrm{NaOH}$ concentrations in the range of 10-15\% to dissolve binder phases and expose the cellulose surface (Chin et al. 2020). On the other hand, mechanical extraction of fibres always leaves some non-cellulosic content in the extracted fibre bundles, while retaining their inherent strength and stiffness in the bamboo culm (Wang and Shao 2020).

Partial hydrolysis in $\mathrm{H}_{2} \mathrm{SO}_{4}$ was used to create natural anchorage mechanisms (microfibrils) in the surface of the fibres. The acid concentration was varied between $20-60 \%$ v/v $\mathrm{H}_{2} \mathrm{SO}_{4}$ with the objective of investigating the effect of external fibrillation over a wide range of concentrations. The effect of this treatment is shown in Fig. 2c-f. Fibrillation is a result of the removal of amorphous cellulose on the fibre surface (Zhao et al. 2007), leaving behind highly crystalline fibres. Hydrolysis preferentially occurs in the amorphous regions, where the acid dissolves amorphous cellulose. At acid concentrations $>40 \%$, microfibrils become evident on the surface of the fibres, shown in white circles in Fig. 2e-f. The fibre diameters were measured from SEM micrographs using the image processing software ImageJ, using approximately 100 measurements/treatment. For acid concentrations $\leq 55 \%$, the distribution of fibre diameters was normal, with a mean and standard deviation of $11 \pm 3 \mu \mathrm{m}$, indicating that the fibrillation was largely a surface reaction. However, at $60 \% \mathrm{v} / \mathrm{v}$ $\mathrm{H}_{2} \mathrm{SO}_{4}$, fibres had begun to degrade, shown by swelling and rupture instances in Fig. $2 \mathrm{~g}-\mathrm{h}$. These observations are in broad agreement with previous 


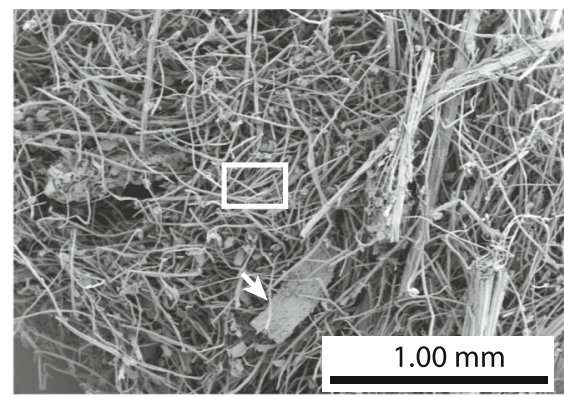

(a)

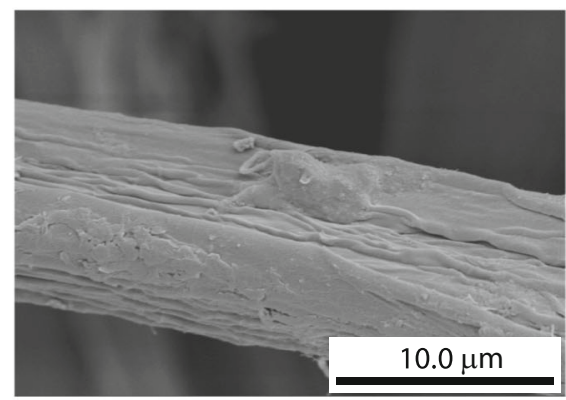

(c)

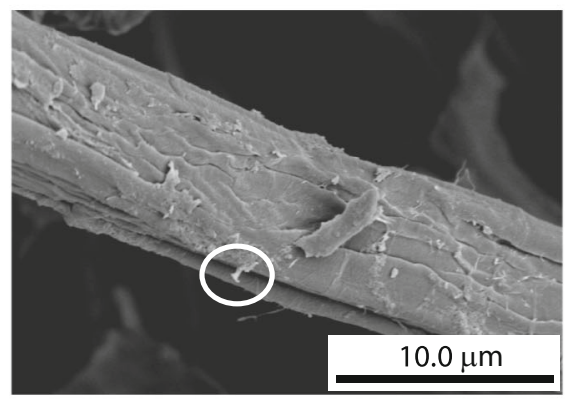

(e)

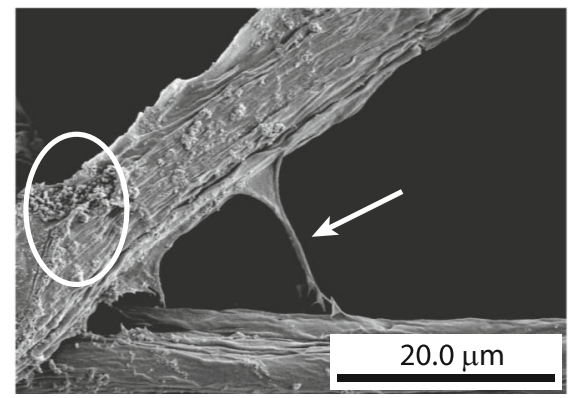

(g)

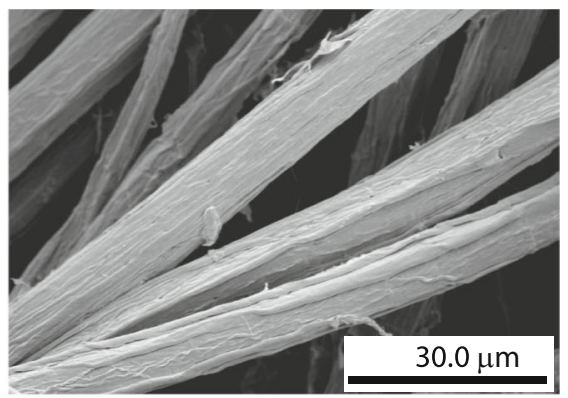

(b)

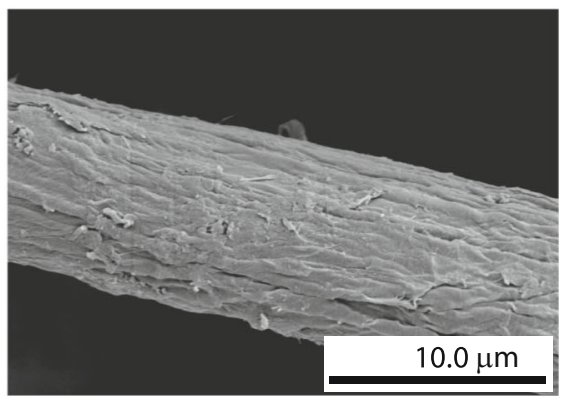

(d)

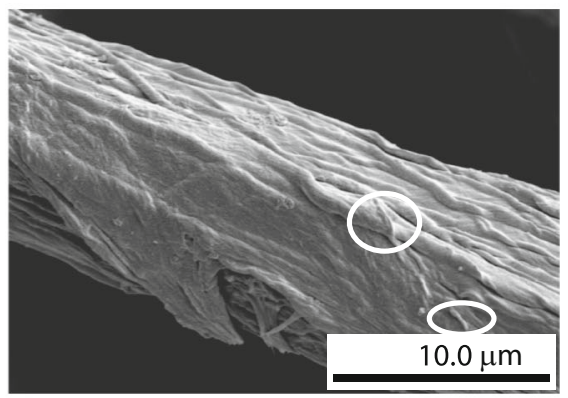

(f)

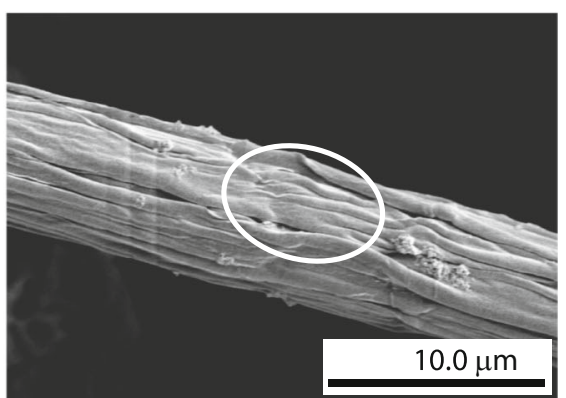

(h)

Fig. 2 Fibre morphology: post-delignification treatment (a), (b), and after partial hydrolysis in $\mathrm{H}_{2} \mathrm{SO}_{4}$ with concentrations $20 \%$ (c), $30 \%(\mathbf{d}), 40 \%(\mathbf{e}), 50 \%(\mathbf{f}), 60 \% \mathrm{v} / \mathrm{v}(\mathbf{g}, \mathbf{h})$

works, e.g. in wheat grass, cellulose hydrolysis in acid concentrations $45-65 \%$ results in highly crystalline cellulose, whereas greater concentrations would cause structural degradation (Huntley et al. 2015). For this reason, $\mathrm{H}_{2} \mathrm{SO}_{4}$ concentrations in the range of $45-55 \%$ were chosen for the fabrication of the fibrous network. 
Foam morphology

The morphology of fibrous networks is shown in Fig. 3a-b. It is composed of fibres with random orientations. The junctions are formed by fibre intertwining, but there is evidence that the external surface fibrillation creates mechanical joints. The surface of cellulose fibres in water is composed of a soft hydrogel layer (Pelton 1993), which enables mutual migration of cellulosic polymers into the opposing surface. The interdiffusion of these molecules occurs through surface capillary junctions, created by the nanofibril "bridges" (Fig. 3c). Increasing the contact area available for the creation of these bonding forces has been reported as one of the key mechanisms for improving the strength of cellulose fibre assemblies (Hirn and Schennach 2015; Thomson et al. 2008).
Figure $3 \mathrm{~d}-\mathrm{e}$ show also the presence of interlocking joints "glued" by cellulose bonds, created during stirring, centrifuging and drying, as capillary forces attract surfaces and adhere them together (Schmied et al. 2013). Consequently, a porous structure of interconnected fibres is formed without requiring the addition of a cross-linker.

The density of the networks was determined through hydrostatic weighing. The specimens were weighed in air, insulated with a thin plastic membrane to prevent the permeation of water, and submerged in a beaker to record the volume difference. The relative density is calculated by $\bar{\rho}=\rho / \rho_{S}$, where $\rho$ is the absolute density of the network, and $\rho_{S}$ is the density of cellulose fibres $\left(1.5 \mathrm{~g} \mathrm{~cm}^{-3}\right.$ (Gibson and Ashby 1997)). Table 1 shows the dependence of the network density on two processing variables: extent of external
Fig. 3 Fibrous network morphology (a, b), and junctions formed by fibril bridges (c) and interlocking joints $(\mathbf{d}, \mathbf{e})$

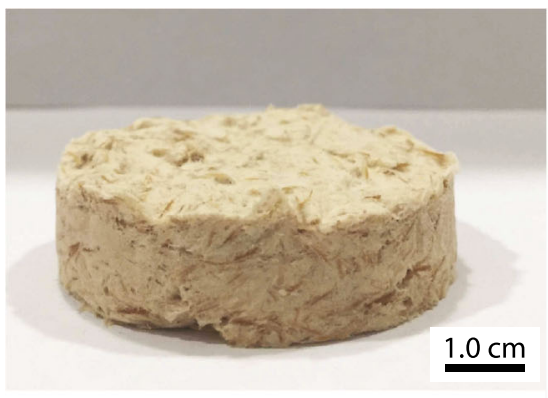

(a)

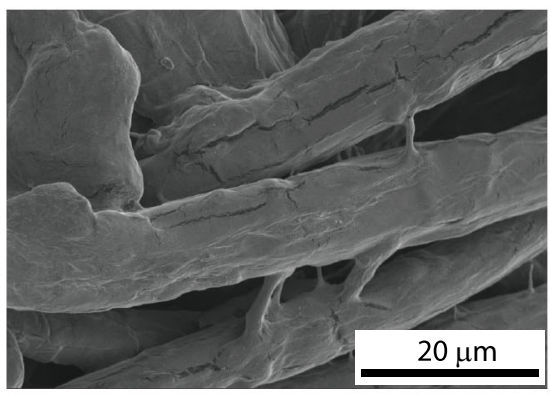

(c)

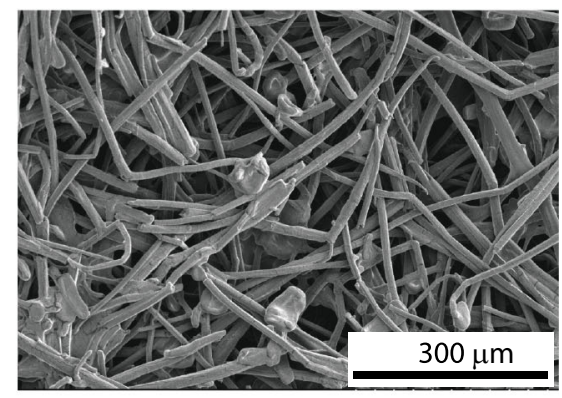

(b)

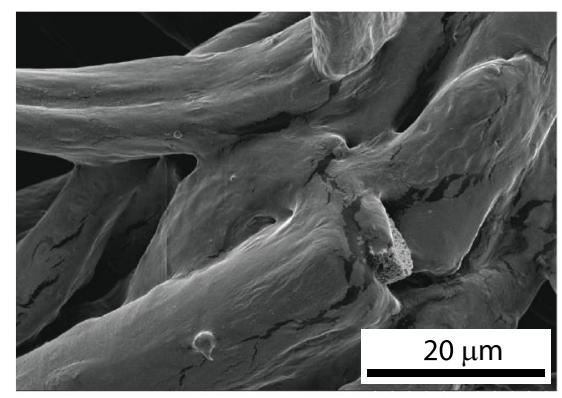

(d)

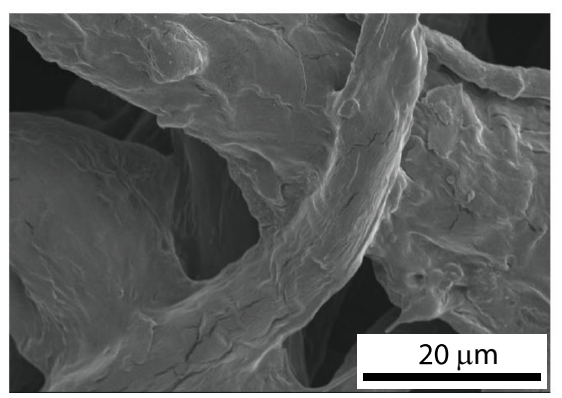

(e) 
Table 1 Physical properties of fibrous networks

\begin{tabular}{llllll}
\hline & $\mathrm{F} 1.1$ & $\mathrm{~F} 1.2$ & $\mathrm{~F} 1.3$ & $\mathrm{~F} 2.1$ & $\mathrm{~F} 3.1$ \\
\hline $\mathrm{H}_{2} \mathrm{SO}_{4}(\mathrm{v} / \mathrm{v})$ & $45 \%$ & $50 \%$ & $55 \%$ & $45 \%$ & $45 \%$ \\
Fibre Length $(\mu \mathrm{m})$ & $500-850$ & $50-850$ & $50-850$ & $850-1000$ & $1000-1700$ \\
Mean Free Length $(\mu \mathrm{m})$ & $130 \pm 30$ & $90 \pm 20$ & $80 \pm 20$ & $150 \pm 10$ & $170 \pm 20$ \\
Density $\left(\mathrm{g} / \mathrm{cm}^{3}\right)$ & $0.45 \pm 0.02$ & $0.89 \pm 0.01$ & $0.94 \pm 0.01$ & $0.32 \pm 0.02$ & $0.24 \pm 0.01$ \\
Relative Density & $0.28 \pm 0.01$ & $0.55 \pm 0.01$ & $0.58 \pm 0.01$ & $0.20 \pm 0.02$ & $0.14 \pm 0.01$ \\
\hline
\end{tabular}

fibrillation (indicated by $\mathrm{H}_{2} \mathrm{SO}_{4}$ concentration; specimens F1.1-F1.3), and fibre length (specimens F1.1F3.1).

Hydrolysis at higher $\mathrm{H}_{2} \mathrm{SO}_{4}$ concentration contributes to increasing the density of the network. An indication of the influence of fibrillation on the number of joints created per unit length of fibre can be obtained by considering the mean free span of fibres, defined as the mean distance of fibres between joints. This was obtained by image analysis and is shown in Table 1. The theoretical relationship between mean free length $\lambda$ and relative network density $\bar{\rho}$ is (Komori and Makishima 1977):

$\lambda=\frac{\pi D}{8 \bar{\rho} J}$

where $D$ is the fibre diameter, and $J$ is the orientation density function. The inverse relationship between mean fibre length and density is generally observed in these networks, indicating that the higher extent of fibrillation is responsible for the increased fibre packing. The effect of initial fibre length on the network density and fibre mean free span is also shown in Table 1. Longer fibres produce networks of lower relative density, with a larger free span.
The morphology of the close-celled foam is shown in Fig. 4. It is composed of inter-penetrating voids, creating a porous structure which is similar to that found in naturally occurring porous foams, e.g. zirconia and mullite (Gibson and Ashby 1997), and polymeric foams, e.g. polyether and whipped mayonnaise suspensions (Gibson and Ashby 1997; Szczurek et al. 2013). The major pores be approximated as triaxial ellipsoids with the major axis parallel to the draining direction (axis 3 in Fig. 4). Their intersections create secondary planar windows of circular crosssection. These features are created during the aeration of the fibre pulp suspension, and are typical of similar structures in e.g. polymerised high internal phase emulsions (Szczurek et al. 2013). Their characteristic dimensions were obtained from SEM micrographs, using standard stereolithography techniques. To account for the difference between the micrograph plane and the plane of maximum cross-section, the mean cell diameters $\left(\overline{L_{i}}\right)$ were evaluated by (DeHoff and Rhines 1968):

$\overline{L_{i}}=\frac{\pi}{2 \bar{Z}_{i}}, i=1,2,3$

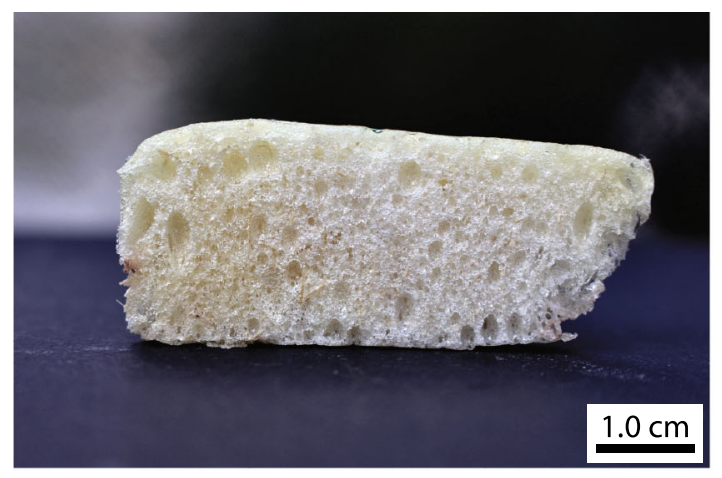

(a)

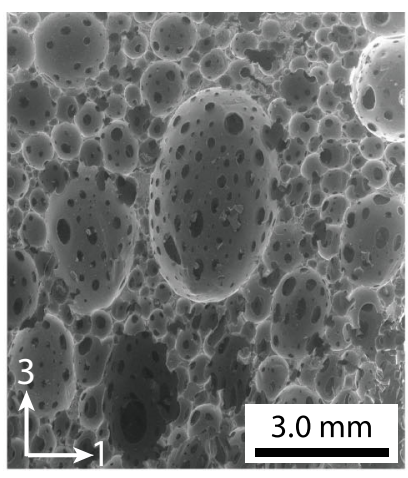

(b)

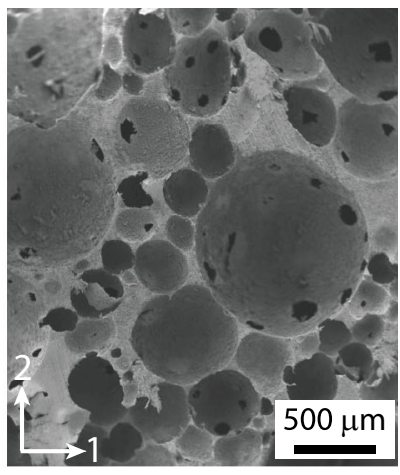

(c)

Fig. 4 Close-celled foam morphology 
where $\overline{Z_{i}}$ is the average value of $Z_{i}$, the reciprocal of the diameter in direction $i$; these values are shown in Table 2. Generally, the cross-sections were observed to be circular in the 12 plane, with $\overline{L_{1}} \approx \overline{L_{2}}$. The aspect ratio of the pores was thus estimated by $R_{31}=\overline{L_{3}} / \overline{L_{1}}=1.49$. The density of these foams was measured with the hydrostatic weighing technique outlined above, and was found to be $\rho=0.21 \pm 0.01$ $\mathrm{g} / \mathrm{cm}^{3}$. Since the density of the cell wall material is unknown, the relative density was instead estimated by the solid area fraction in the SEM micrographs, with a mean value $\bar{\rho}=0.20 \pm 0.02$. We note that the close cell foams achieve a substantially lower density compared to their fibrous network counterparts.

\section{Mechanical response}

The compressive response of both types of foams investigated in this study shown in Fig. 5. We start by discussing the response of close-celled foams, shown in Fig. 5a. Due to the orthotropic morphology of the internal structure, specimens of these foams were tested in uniaxial compression along the three orthogonal axes of Fig. 3, to investigate the directional sensitivity of mechanical properties. The compressive response is typical of elastic-brittle foams, with three distinct regimes: a linear elastic regime, plateau (with the typical load drops of brittle cell crushing), and densification (Gibson and Ashby 1997). The mechanical properties of interest are the elastic modulus $(E)$, crushing strength $\left(\sigma_{C R}\right)$, and energy absorption to densification, defined here as:

$$
U_{D, i}=\int_{0}^{\varepsilon_{D}} \sigma_{i} d \varepsilon_{i}
$$

where $i$ is the direction of uniaxial stress, and $\varepsilon_{D}$ is the densification strain, defined here as the strain value

Table 2 Physical properties of close-celled foams

\begin{tabular}{ll}
$\overline{L_{1}}, \overline{L_{2}}(\mathrm{~mm})$ & $1.15 \pm 0.65$ \\
$\overline{L_{3}}(\mathrm{~mm})$ & $1.72 \pm 0.50$ \\
$R_{31}$ & 1.49 \\
Density $\left(\mathrm{g} / \mathrm{cm}^{3}\right)$ & $0.21 \pm 0.01$ \\
Relative Density & $0.20 \pm 0.02$ \\
\hline
\end{tabular}

where the stress increases above the maximum stress in the plateau regime, as indicated in Fig. 5a. A summary of these properties is shown in Table 3 . The response is slightly stiffer (by 20\%) in the pore rise direction, however the crushing strength, densification strain, and energy absorbed to densification are practically identical. This is in agreement with theoretical predictions of the stiffness and strength of brittle close-celled foams (Gibson and Ashby 1997). Generally the stiffness is more sensitive to anisotropy than crushing strength, e.g. ideal close-celled foams with uniform pore aspect ratio $\mathrm{R}=1.5$ and with $50 \%$ material volume fraction contained in the cell edges would have predicted stiffness ratio of 3 and strength ratio of 1.6 (Gibson and Ashby 1997). The directionality effect observed here is more subdued due to three deviations from the theoretical model of ideal foams: interpenetrating cells, non-uniform pore aspect ratio, and misalignment of the pore major axis with the direction of compression.

Next, we discuss the deformation mechanisms of these foams. Figure 6 shows micrographs of collapse mechanisms in close-celled foams loaded along the 3 direction, at macroscopic strains $\varepsilon_{3}=0.1$ (a) and 0.4 (b). In the initial stages of crushing, the primary deformation mechanism is opening of multiple fracture surfaces in secondary voids, shown in white arrows in Fig. 6a. These micro-cracks subsequently lead to brittle collapse of the cell walls, as shown in Fig. 6 b. At the initiation of densification (i.e. at $\varepsilon_{D} \approx 0.5$ ), the morphology of the porosity is largely retained, with the fracture surface propagating through the secondary pores and densification occurring due to interpenetration of the latter.

The compressive response of the fibrous networks is shown in Fig. 5b. Due to the random distribution of fibres, there was no discernible difference in responses from different testing directions. The response of these foams undergoes two distinct regimes: an initial linear regime, followed gradually by strain stiffening. Unlike close-celled foams, fibre networks do not exhibit a marked transition to the densification regime (Picu 2011; Alimadadi et al. 2018; Chatti et al. 2018; Islam and Picu 2018). We thus define the densification stress and strain $\left(\sigma_{D}\right.$ and $\varepsilon_{D}$ respectively) as the point where the instantaneous modulus takes the value $d \sigma / d \varepsilon=1.5 E$, with $E$ denoting the stiffness of the initial linear regime; these points are depicted in 


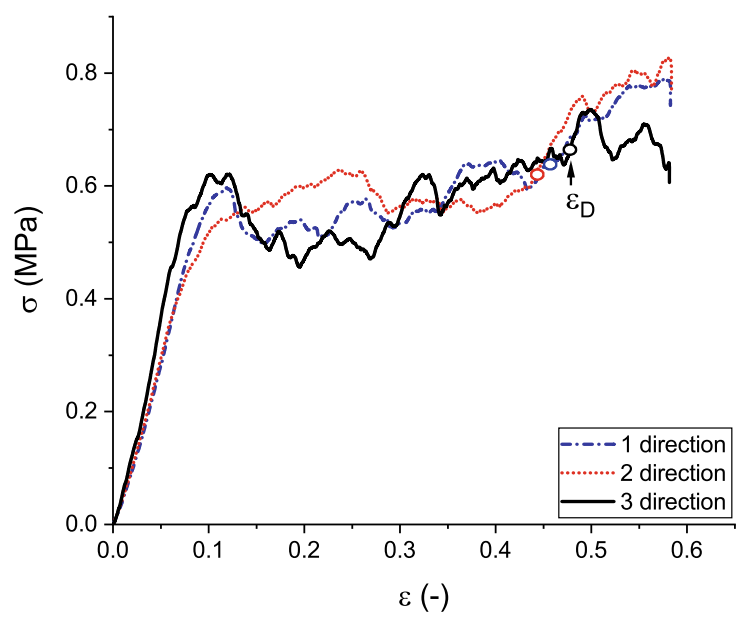

(a)

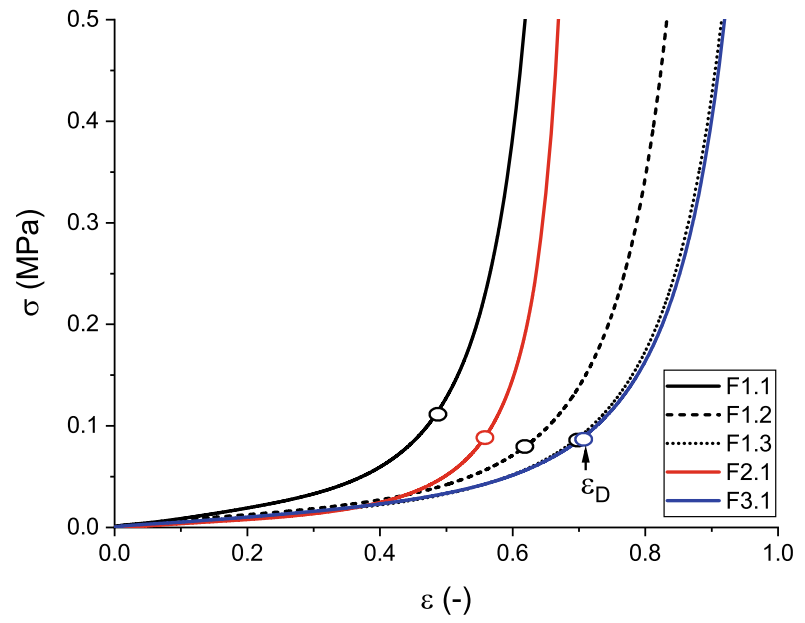

(b)

Fig. 5 Uniaxial compressive response of close-celled foams (a) and fibrous networks (b), showing the start of densification regime in open circles

Table 3 Summary of compressive properties of close-celled foams and fibrous networks

\begin{tabular}{llllll}
\hline Foam & Type/direction & $E(\mathrm{MPa})$ & $\sigma_{C}, \sigma_{D}(\mathrm{MPa})$ & $\varepsilon_{D}$ & $U_{C}, U_{D}\left(\mathrm{~kJ} / \mathrm{m}^{3}\right)$ \\
\hline Closed & 1-direction & $6.2 \pm 0.6$ & $0.48 \pm 0.10$ & 0.53 & $160 \pm 90$ \\
& 2-direction & $6.3 \pm 0.2$ & $0.50 \pm 0.10$ & 0.52 & $190 \pm 50$ \\
& 3-direction & $7.2 \pm 0.5$ & $0.49 \pm 0.06$ & 0.57 & $180 \pm 20$ \\
Fibrous & F1.1 & $0.10 \pm 0.02$ & $0.11 \pm 0.01$ & 0.49 & $16 \pm 3$ \\
& F1.2 & $0.06 \pm 0.01$ & $0.08 \pm 0.01$ & 0.62 & $16 \pm 4$ \\
& F1.3 & $0.05 \pm 0.01$ & $0.08 \pm 0.01$ & 0.69 & $17 \pm 2$ \\
& F2.1 & $0.06 \pm 0.01$ & $0.09 \pm 0.01$ & 0.56 & $11 \pm 2$ \\
& F3.1 & $0.05 \pm 0.01$ & $0.08 \pm 0.01$ & 0.70 & $18 \pm 2$ \\
\hline
\end{tabular}

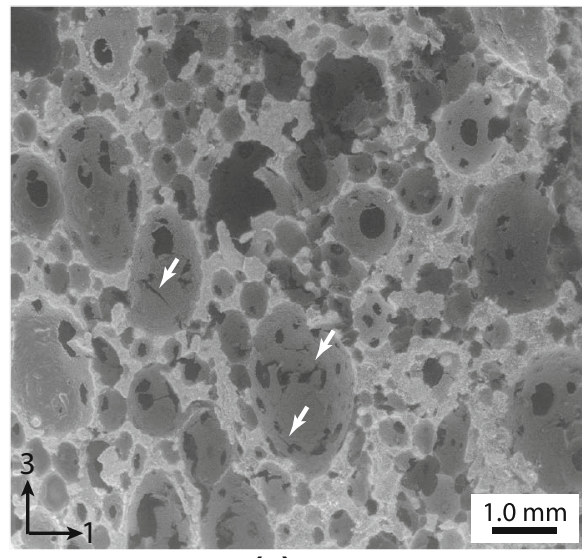

(a)

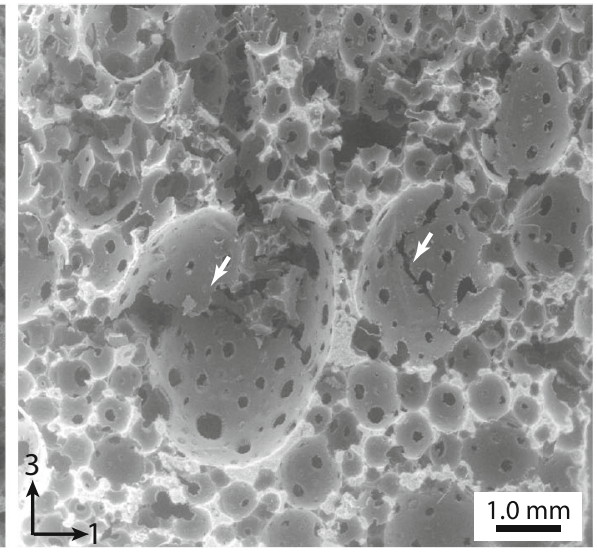

(b)

Fig. 6 Micrographs of fracture locations in close celled bamboo foams at macroscopic compressive strain of 0.1 (a) and 0.4 (b) 
Fig. $5 \mathrm{~b}$ and capture reasonably consistently the initiation of the strain stiffening. The energy absorption to densification, $U_{D}$, is defined as in Eq. 3, and all material properties are summarised in Table 3.

In the literature of fibre network compression, the initial regime is composed of two sub-regions: an initial small-strain linear regime due to fibre rearrangement (Chatti et al. 2018) or bending (Islam et al. 2017), followed by a second regime controlled by fibre bending and buckling (Islam et al. 2017, 2018). The response of the present networks showed no marked distinction between these two sub-regimes; this may be caused by a spatially distributed activation of the above 3 mechanisms, due to the random fibre orientation. The densification regime occurs due to the internal geometrical constraints arising from the development of new fibre-fibre contacts at large strains (Islam et al. 2017, 2018; Alimadadi et al. 2018).

It is noted that increasing the constituent fibre length (specimens F1.1-F3.1) results in a reduction in the compressive modulus, as would be expected from a similar trend in density. Scaling relationships for the stiffness of foams take the form $E / E_{S}=C\left(\rho / \rho_{S}\right)^{n}$ where $E$ and $\rho$ are the stiffness and density of the foam, $E_{S}$ and $\rho_{S}$ are the stiffness and density of the constituent solid (in this case we use cellulose $E_{S}=$ $25 \mathrm{GPa}$, and $\rho_{S}=1.5 \mathrm{~g} / \mathrm{cm}^{3}$ (Gibson and Ashby 1997), and $C$ and $n$ are non-dimensional scaling parameters. Gibson and Ashby collected the form of these scaling laws for many cellular solids, where they reported a range from 1 to 3 for the exponent $n$. The value of this exponent is an indication both of the connectivity of the network, and the mechanisms that dictate its deformation (e.g. bending or stretch-dominated) (Deshpande et al. 2001). The mechanics of fibre networks indicate that in low-density networks $E$ scales with $\rho^{2}$; this corresponds to non-affine deformation, and fibres that deform primarily by bending (Shahsavari and Picu 2012; Licup et al. 2016). Conversely, in large density networks, $E$ scales with $\rho$; these networks exhibit affine deformation, and fibres that store strain energy by stretching. In the present bamboo fibre networks, we observe $n=1.2$, which suggests a stretch-dominated deformation mechanism in the initial linear regime. As the deformation progresses to densification, the effect of a higher initial fibre length is in increasing the densification strain at a lower flow stress, thus no discernible difference is seen in the energy absorption to densification.

Finally, we assess the effect of external fibrillation on mechanical properties (specimens F1.1-F1.3). As explained above, a higher extent of fibrillation produces networks with higher density, due to the increase of the joint density/fibre length. Interestingly, the compressive response shows that this also reduces the initial compressive stiffness, e.g. in the scaling relationship shown above, $n=-1.0$. This unusual scaling relationship is a consequence of the relatively low strength of the microfibril bridges, which act as joints with low compressive and torsional strength. Numerical analyses have shown that networks with rigid fibre-fibre contacts exhibit a higher stiffness than networks without contacts (Alimadadi et al. 2018). We therefore conclude that, while necessary for creating the fibre network without binding agents, the external fibrillation produces structurally efficient networks at lower hydrolysis concentrations.

\section{Concluding remarks}

In this work, cellular solids were manufactured from cellulose fibres extracted from bamboo culms. Two approaches were used, to create fibre networks and solid foams. The fibre networks were created with no binders or blowing agents, relying instead on partial hydrolysis of the extracted cellulose fibres in $\mathrm{H}_{2} \mathrm{SO}_{4}$. The partial hydrolysis produced microfibrils which, during the foaming process, act as bridges and aid the interlocking of fibres. The $\mathrm{H}_{2} \mathrm{SO}_{4}$ concentration determines the extent of fibrillation; SEM micrographs showed a higher external fibrilation and surface degradation at greater concentrations. Higher concentrations produce a tightly packed network, shortening the fibre mean free span and increasing the relative density. However this results in detrimental effects in mechanical response; the initial stiffness generally decreased at higher densities due to the low strength of microfibril bridges. The effect of constituent fibre length was also investigated, and showed that lowdensity networks could be obtained from longer constituent fibres. The initial stiffness of these networks was shown to scale linearly with density, indicating that the network response was dominated by fibre stretching. 
Close-celled foams were created with a simple approach, by using natural foaming agents, binders and surfactants. Their internal microstructure consisted of interpenetrating ellipsoidal voids, with an aspect ratio of 1.5, and the compressive response was typical of elastic-brittle foams. Internal energy absorption was through the mechanism of crack initiation and propagation through the intersection of internal pores. The mechanical response was isotropic in densification strength and energy absorption, but the initial stiffness was higher by $20 \%$ in the pore rise direction. The response of these first cellular materials created from bamboo fibres show that they are within the range of presently available foams, and indicate a potential route towards the manufacturing of sustainable lightweight materials for structural applications.

Acknowledgments The authors are grateful to The Royal Society (Grant Number RG170243), and the Department of Mechanical Engineering, University College London, for the financial support provided for this project.

\section{Compliance with ethical standards}

Conflicts of interest All authors declare that they have no conflict of interest.

Open Access This article is licensed under a Creative Commons Attribution 4.0 International License, which permits use, sharing, adaptation, distribution and reproduction in any medium or format, as long as you give appropriate credit to the original author(s) and the source, provide a link to the Creative Commons licence, and indicate if changes were made. The images or other third party material in this article are included in the article's Creative Commons licence, unless indicated otherwise in a credit line to the material. If material is not included in the article's Creative Commons licence and your intended use is not permitted by statutory regulation or exceeds the permitted use, you will need to obtain permission directly from the copyright holder. To view a copy of this licence, visit http://creativecommons.org/licenses/by/4.0/.

\section{References}

Alimadadi M, Uesaka T (2016) 3d-oriented fiber networks made by foam forming. Cellulose 23(1):661-671

Alimadadi M, Lindström SB, Kulachenko A (2018) Role of microstructures in the compression response of three-dimensional foam-formed wood fiber networks. Soft Matter $14: 8945-8955$

ASTM D1621-16, (2016) Standard test method for compressive properties of rigid cellular plastics. Standard, ASTM International
Bernardin LJ (1969) Process of forming absorbent paper from a mixture of cellulosic fibers and partially crosslinked cellulosic fibers and paper thereof. US Patent 3,837,999

Cervin NT, Andersson L, Ng JBS, Olin P, Bergström L, Wågberg L (2013) Lightweight and strong cellulose materials made from aqueous foams stabilized by nanofibrillated cellulose. Biomacromolecules 14(2):503-511

Chatti F, Poquillon D, Bouvet C, Michon G (2018) Numerical modelling of entangled carbon fibre material under compression. Comp Mater Sci 151:14-24

Chin SC, Tee KF, Tong FS, Ong HR, Gimbunh J (2020) Thermal and mechanical properties of bamboo fiber reinforced composites. Mater Today Comm 23:100876

Chung R (1974) Method of controlling the orientation of fibers in a foam formed sheet. US Patent 3,837,999

Correia V, Curvelo A, Marabezi K, Almeida A, Savastano H (2015) Bamboo cellulosic pulp produced by the ethanol/ water process for reinforcement applications. Cienc Florest 25(1):127-135

DeHoff R, Rhines FN (1968) Quantitative microscopy. McGraw-Hill, New York

Demitri C, Giuri A, Raucci M, Giuliano D, Madaghiele M, Sannino A, Ambrosio L (2014) Preparation and characterization of cellulose-based foams via microwave curing. Interface Focus 4:20130053

Deshpande VS, Ashby MF, Fleck NA (2001) Foam topology: bending versus stretching dominated architectures. Acta Mater 49(6): 1035-1040

Fabiyi J, Ogunleye B (2015) Mid-infrared spectroscopy and dynamic mechanical analysis of heat-treated obeche (triplochiton scleroxylon)wood. Mad Cienc Tech 17(1):5-16

Faix O (1991) Classification of lignins from different botanical origins by ft-ir spectroscopy. Holzforschung 45:21-27

Ferreira ES, Rezende CA (2018) Simple preparation of cellulosic lightweight materials from eucalyptus pulp. ACS Sustain Chem Eng 8(11):14365-14373

Gibson LJ, Ashby MF (1997) Cellular solids: structure and properties. Cambridge University Press, Cambridge

Härkäsalmi T, Lehmonen J, Itälä J, Peralta C, Siljander S, Ketoja J (2017) Design-driven integrated development of technical and perceptual qualities in foam-formed cellulose fibre materials. Cellulose 24(11):2039-2045

Harris MA, Koomson CK (2015) Cold-water in situ dissolution of bamboo starch in a bioreactor: inducing forest recovery from an anthropogenic disturbance. Int J Env Sci Tech 12:2503-2510

Hirn U, Schennach R (2015) Comprehensive analysis of individual pulp fiber bonds quantifies the mechanisms of fiber bonding in paper. Sci Rep 5(10503):1-4

Huntley C, Crews K, Abdalla M, Russell A, Curry M (2015) Influence of strong acid hydrolysis processing on the thermal stability and crystallinity of cellulose isolated from wheat straw. Int J Chem Eng 2015:1-11

Islam MR, Picu RC (2018) Effect of network architecture on the mechanical behavior of random fiber networks. J App Mech 85(8):081011

Islam MR, Tudryn G, Bucinell R, Schadler L, Picu RC (2017) Morphology and mechanics of fungal mycelium. Sci Rep $7: 13070$ 
Islam MR, Tudryn G, Bucinell R, Schadler L, Picu RC (2018) Stochastic continuum model for mycelium-based biofoam. Mater Des. 160:549-556

Johar N, Ahmad I, Dufresne A (2012) Extraction, preparation and characterization of cellulose fibres and nanocrystals from rice husk. Ind Crop Prod 37:93-99

Kang J, Cho G, Kwon Y (2012) An innovative foam insulation produced from cellulose. Proc Korea Inst Const Tech Build Encl Tech Envt Council 1-8

Ketoja JA, Paunonen S, Jetsu P, Pääkkönen E (2019) Compression strength mechanisms of low-density fibrous materials. Mater-Basel 12(3):384

Kinnunen K, Lehmonen J, Beletski N, Jetsu P, Hjelt T (2013) Benefits of foam forming technology and its applicability in high mfc addition structures. In: Proceedings 15th funds restricting symposium pp 837-850

Komori T, Makishima K (1977) Numbers of fiber-to-fiber contacts in general fiber assemblies. Text Res J 47:13-17

Leavitt S, Danzer S (1993) Method for batch processing small wood samples to holocellulose for stable-carbon isotope analysis. Anal Chem 65(1):87-88

Licup AJ, Sharma A, MacKintosh FC (2016) Elastic regimes of subisostatic athermal fiber networks. Phys Rev E 93(1):012407. https://doi.org/10.1103/PhysRevE.93. 012407

Lima M, Lavorente G, da Silva H, Bragatto J, Rezende C, Bernardinelli O, deAzevedo E, Gomez L, McQueen-Mason S, Labate C, Polikarpov I (2013) Effects of pretreatment on morphology, chemical composition and enzymatic digestibility of eucalyptus bark: a potentially valuable source of fermentable sugars for biofuel production - part 1. Biotech Biofuel 6(75):1-17

Madani A, Zeinoddini S, Varahmi S, Turnbull H, Phillion AB, Olson JA, Martinez DM (2014) Ultra-lightweight paper foams: processing and properties. Cellulose 21:2021-2031

Merle J, Birot M, Deleuze H, Mitterer C, Carré H, Bouhtoury FCE (2016) New biobased foams from wood byproducts. Mater Des. 91:192-196

Miltz J, Ramon O (1990) Energy absorption characteristics of polymeric foams used as cushioning materials. Polym Eng Sci 30(2):129-133

Mimin V, Kabrelian V, Fackler K, Hettegger H, Potthast A, Rosenau T (2016) Lignin-based foams as insulation materials: a review. Holzforschung 73(1):117-130

Mohamed A, Hassabo A (2015) Flame retardant of cellulosic materials and their composites. Flame Ret. https://doi.org/ 10.1007/978-3-319-03467-6_10

Moradbak A, Tahir P, Mohamed A, Halis R (2015) Alkaline sulfite anthraquinone and methanol pulping of bamboo (gigantochloa scortechinii). Bio Res 11(1):240

Ottenhall A, Seppän T, Ek M (2018) Water-stable cellulose fiber foam with antimicrobial properties for bio based lowdensity materials. Cellulose 25:2599-2613

Pelton R (1993) A model of the external surface of wood pulp fibers. Nord Pulp Pap Res J 8:113-119

Picu RC (2011) Mechanics of random fiber networks - a review. Soft Matter 7:6768-6785

Poehler T, Jetsu P, Fougeron A, Barraud V (2017) Use of papermaking pulps in foam-formed thermal insulation materials. Nord Pulp Paper Res J 32(3):367-374
Prasad S, Singh A, Joshi HC (2007) Ethanol as an alternative fuel from agricultural, industrial and urban residues. Resour Conserv Recy 50(1):1-39

Radvan B (1964) Basic radfoam process. British Patent 1329409

Rapp F, Schneider A, Elsner P (2014) Biopolymer foams relationship between material characteristics and foaming behavior of cellulose based foams. In: AIP Conference Proceeding pp 361-366

Reddy KO, Shukla M, Maheswari CU, Rajulu AV (2012) Mechanical and physical characterization of sodium hydroxide treated Borassus fruit fibers. J Forestry Res 23(4):667-674

Saz-Orozco BD, Alonso M, Oliet M, Domínguez J, Rodriguez F (2013) Effects of formulation variables on density, compressive mechanical properties and morphology of wood flour-reinforced phenolic foams. Compos Part B-Eng 56:546-552

Schmied F, Teichert C, Kappel L, Hirn U, Bauer W, Schennach R (2013) What holds paper together: Nanometre scale exploration of bonding between paper fibres. Sci Rep 3:1-6

Sfiligoj M, Hribernik S, Stana K, Kree T (2013) Plant fibres for textile and technical applications. Intech Open Ltd, London

Shahsavari A, Picu RC (2012) Model selection for athermal cross-linked fiber networks. Phys Rev E 86(1):011923. https://doi.org/10.1103/PhysRevE.86.011923

Stevens ES, Klamczynski A, Glenn GM (2010) Starch-lignin foams. Express Polym Lett 4(5):311-320

Svagan A, Berglund L, Jensen P (2011) Cellulose nanocomposite biopolymer foam-hierarchical structure effects on energy absorption. ACS Appl Mater Inter 3:1411-1417

Szczurek A, Fierro V, Pizzi A, Celzard A (2013) Mayonnaise, whipped cream and meringue, a new carbon cuisine. Carbon 58:245-248

Szczurek A, Fierro V, Pizzi A, Stauber M, Celzard A (2014) A new method for preparing tannin-based foams. Ind Crop Prod 54:40-53

Thomson C, Lowe R, Ragauskas A (2008) First characterization of the development of bleached kratf softwood pulp fiber interfacesduring drying and rewetting using fret technology. Holzforschung 62:383-388

Tondi G, Pizzi A (2009) Tannin-based rigid foams: Characterization and modification. Ind Crop Prod 29:356-363

Tondi G, Link M, Kolbitsch C, Gavino J, Luckeneder P, Petutschnigg A, Herchl R, Doorslaer CV (2016) Lignin-based foams: Production process and characterization. Bio Res 11(2):2972-2986

Tu Y, Suppes G, Hsieh F (2008) Water-blown rigid and flexible polyurethane foams containing epoxidized soybean oil triglycerides. J App Polym Sci 109(1):537-544

Wang F, Shao Z (2020) Study on the variation law of bamboo fibers' tensile properties and the organization structure on the radial direction of bamboo stem. Ind Crop Prod 152:112521. https://doi.org/10.1016/j.indcrop.2020.112521

Zhao H, Kwak JH, Zhang ZC, Brown HM, Arey BW, Holladay JE (2007) Studying cellulose fiber structure by sem, xrd, nmr and acid hydrolysis. Carbohydr Polym 68(2):235-241

Publisher's Note Springer Nature remains neutral with regard to jurisdictional claims in published maps and institutional affiliations. 